\title{
Variabilidad de presión de pulso y pletismográfica en pacientes con enfermedad renal crónica e implante renal bajo anestesia general equilibrada
}

\section{Pulse pressure variability and plethysmographic variability in patients with chronic kidney disease and elective kidney implant placement under balanced general anesthesia}

\author{
Diego Escarramán-Martínez ${ }^{1,2}$, Martha A. Montes-Alvarado ${ }^{3}$, Alejandro Lima-Molina ${ }^{2,3}$, \\ Álvaro J. Montiel-Jarquín ${ }^{2,3 *}$ y Arturo García-Galicia ${ }^{2,3}$ \\ ${ }^{1}$ Unidad Médica de Alta Especialidad, Hospital de Especialidades, Centro Médico Nacional La Raza, Instituto Mexicano del Seguro Social, Ciudad de \\ México; ${ }^{2}$ Facultad de Medicina, Universidad Popular Autónoma del Estado de Puebla, Puebla, Puebla; ${ }^{3}$ Unidad Médica de Alta Especialidad, Hospital de \\ Especialidades de Puebla, Centro Médico Nacional Gral. de División Manuel Ávila Camacho, Instituto Mexicano del Seguro Social, Puebla, Puebla. México
}

\section{Resumen}

Introducción: El monitoreo hemodinámico dinámico es fundamental en quirófano para tomar decisiones de fluidoterapia encaminadas a disminuir complicaciones en pacientes sometidos a cirugía. Material y métodos: Estudio descriptivo. Se recolectó el registro de la variabilidad de presión de pulso $(d P P)$ y el índice de variabilidad pletismográfica (PVi) durante la primera hora de cirugía. Las variables con distribución normal se describen con media aritmética y desviación estándar, y aquellas que no presentan distribución normal con mediana y rango intercuartílico. La prueba de contraste de hipótesis de datos discretos fue la t de Student de muestras independientes. Para medir la concordancia se construyó un polar plot; para cuantificar su validez se calculó la razón de verosimilitud positiva/negativa y se graficó un nomograma de Fagan. Resultados: De 11 pacientes estudiados 8 fueron de sexo masculino y 3 de sexo femenino. El polar plot mostró concordancia casi perfecta entre ambos métodos. Razón de verosimilitud positiva de 2.35 y negativa de 0.1. El normograma de Fagan dio como resultado una probabilidad postest positiva del 97\%. Conclusiones: La concordancia y la validez entre los resultados del monitoreo hemodinámico dinámico no invasivo (PVi) e invasivo (dPP), son casi perfecta y excelente, respectivamente.

Palabras clave: Variabilidad de presión de pulso. Índice de variabilidad pletismográfica. Enfermedad renal crónica. Anestesia general equilibrada. Monitoreo hemodinámico dinámico.

\section{Abstract}

Introduction: dynamic hemodynamic monitoring is essential in the operating room to make fluid therapy decisions aimed at reducing complications in patients undergoing surgery. Material and methods: descriptive study. The record of pulse pressure variability $(d P P)$ and plethysmographic variability $(P V i)$ was collected during the first hour of surgery. Variables with normal distribution are described with arithmetic mean and standard deviation, while those that do not present normal distribution with median and interquartile range. The discrete data hypothesis contrast test was the Student's test for independent samples. To measure agreement, a Polar Plot was constructed, while, to quantify its validity, the positive/negative likelihood

Correspondencia:

*Álvaro J. Montiel-Jarquín E-mail: dralmoja@ hotmail.com
Disponible en internet: 17-12-2021 Rev Hosp Jua Mex. 2021;88(4):164-169

www.revistahospitaljuarez.com 1405-9622/@ 2020 Sociedad Médico-Quirúrgica del Hospital Juárez de México, A.C. Publicado por Permanyer. Este es un artículo open access bajo la licencia CC BY-NC-ND (http://creativecommons.org/licenses/by-nc-nd/4.0/). 
ratio was calculated and a Fagan nomogram was plotted. Results: of 11 patients studied, 8 were male and 3 females. The polar plot showed almost perfect agreement between both methods. The positive likelihood ratio of 2.35 and negative 0.1 . Fagan's normogram resulted in a positive post-test probability of $97 \%$. Conclusions: the concordance and validity between the results of the non-invasive dynamic hemodynamic monitoring PVi and invasive $d P P$, are almost perfect and excellent respectively.

Key words: Pulse pressure variability. Plethysmographic variability index. Chronic kidney disease. Balanced general anesthesia. Dynamic hemodynamic monitoring.

\section{Introducción}

El mantenimiento de la volemia, la homeostasis de los líquidos y evitar la sobrecarga hídrica es muy importante en los pacientes con enfermedad renal crónica sometidos a anestesia general equilibrada ${ }^{1}$. La fluidoterapia ha tenido variantes desde que Shoemaker, et al. en 1980 propusieron los valores fisiológicos supranormales como metas en el monitoreo hemodinámico en pacientes críti$\cos ^{2}$; desde entonces, se han ocupado diferentes variables, pasando por la presión venosa central, el gasto urinario, el gasto cardiaco y la saturación parcial de oxígeno, para el monitoreo hemodinámico. Actualmente, se utilizan los parámetros hemodinámicos dinámicos como parte del manejo de la terapia guiada por objetivos (GDT), los cuales dependen de las interacciones cardiopulmonares en pacientes bajo anestesia general y ventilación mecánica ${ }^{3}$. La evolución del monitoreo hemodinámico ha ido desde dispositivos totalmente invasivos, pasando por dispositivos mínimamente invasivos, hasta dispositivos no invasivos, con lo que se erradican complicaciones relacionadas con mecanismos invasivos, como colocación de catéteres, tanto centrales como arteriales periféricos o del tipo Swan-Ganz ${ }^{4}$.

Los parámetros dinámicos de la respuesta a fluidos se relaciona con las interacciones cardiopulmonares en pacientes bajo anestesia general con ventilación mecánica; su ventaja radica en que se pueden obtener por medio de una simple onda de presión arterial, la variabilidad de presión de pulso (dPP) ${ }^{5}$. La dPP es la diferencia entre la presión de pulso (PP) máxima y mínima durante el ciclo respiratorio dividida por el promedio de esta:

$\mathrm{dPP}=\mathrm{dPP}$ máx $-\mathrm{dPP}$ mín/(dPP máx + dPP mín/2)

Cuando la dPP se mide como la diferencia entre la presiónarterial sistólica(PAS) y lapresión arterial diastólica del latido anterior, es una medida directamente proporcional al volumen sistólico (VS) ${ }^{6}$.

La oximetría de pulso estándar utiliza dos longitudes de onda de luz en el espectro rojo (R) e infrarrojo (IR), la absorbancia de la señal IR no se ve afectada por los cambios en la saturación arterial de oxígeno y cambia con las pulsaciones asociadas con el volumen de sangre en el lecho vascular periférico en el sitio del sensor. La forma de onda de la fotopletismografía mostrada en algunos oxímetros de pulso representa el componente pulsátil altamente procesado y filtrado de la señal IR a lo largo del tiempo; entonces, las variaciones respiratorias en la amplitud de la forma de onda ( $\triangle \mathrm{POP}$ ) se parecen a los cambios cíclicos en la presión arterial sistémica y el VS que ocurren durante el ciclo respiratorio medidos con un catéter arterial ${ }^{7}$. El PVi se calcula como la diferencia entre los valores máximo y mínimo del índice de perfusión (PI) (relación entre la absorción de luz infrarroja pulsátil y no pulsátil) durante un ciclo respiratorio dividida por el PI máximo8:

$$
\text { PVi = (PImáx - PImín/PImáx) x } 100
$$

El índice de variabilidad pletismográfica (PVi) es el primer índice disponible comercialmente que calcula de forma automática y continua las variaciones respiratorias en la fotopletismografía a partir de datos recopilados de forma no invasiva por medio de un sensor de pulsioximetría y se correlaciona visualmente con la $\triangle P O P$ con el uso de un algoritmo diferente para su cálculo, basado en el PI y es totalmente no invasivo ${ }^{9,10}$.

\section{Objetivo}

Determinar y examinar la posibilidad de sustituir el dPP por el PVi durante cirugía de implante renal bajo anestesia general equilibrada, midiendo la concordancia y validez de ambos métodos de monitoreo hemodinámico.

\section{Material y métodos}

Estudio, longitudinal, en el que se estudiaron 11 pacientes con enfermedad renal crónica sometidos de forma electiva a cirugía de implante renal bajo anestesia general equilibrada. Para la inducción anestésica se utilizó fentanilo $3-5 \mathrm{mcg} / \mathrm{kg}$, cisatracurio $0.1 \mathrm{mg} / \mathrm{kg}$, propofol $1-2 \mathrm{mg} / \mathrm{kg}$ y lidocaína $1-1.5 \mathrm{mg} / \mathrm{kg}$; el mantenimiento se realizó con sevoflorano $1 \mathrm{CAM}$. Inmediatamente después de la inducción, se realizó prueba de Allen, se canalizó 
la arteria radial, para conectar a un monitor de la máquina de anestesia GE Healtcare ${ }^{\circledR}$ modelo Aspire para monitoreo continuo de dPP, colocación de un monitor MyghtySat ${ }^{\circledR}$ para monitoreo continuo del $\mathrm{PVi}$, con el registro de los valores cada 15 minutos durante la primera hora de la cirugía para tener una relajación neuromuscular buena.

Para la exploración de la validez, se tomó como monitor gold standard la dPP con un punto de corte < 12 y la prueba del PVi como monitoreo para este, para determinar cuál es el punto de corte mejor.

Parala estadística descriptiva, primero se verificóla normalidad, las variables con distribución normal se describieron con medias como medida de tendencia central y desviaciónestándar(DE) comodispersión; aquellassin distribución normal se describieron con mediana como medida de tendencia central y rango intercuartílico (RIQ) como posición relativa.

Para la construcción del polar plot primero se organizaron los datos en dos columnas: $X$ para el método de referencia (dPP) e Y para el método de prueba (PVi), posterior se convirtieron los datos estáticos de los monitores a datos dinámicos (cambios), con la siguiente fórmula: $X_{2}-X_{1} \ldots$ y $Y_{2}-Y_{1}$ y así sucesivamente con todos los datos, obteniendo $\Delta \mathrm{X}$ y $\Delta \mathrm{Y}$. Con este primer paso se obtuvieron las coordenadas cartesianas. Para su transformación en coordenadas polares primero se calculó la hipotenusa (r) con la raíz cuadrada del cuadrado de $\Delta \mathrm{X}$ y $\Delta \mathrm{Y}$; por último, se calculó el ángulo theta con la función inversa de tangente $(\mathrm{Y} / \mathrm{X})$.

Se exploró la diferencia entre las observaciones de las variables discretas dPP y PVi por la prueba $t$ de Student de muestras independientes tomando un valor de $p<0.05$ como estadísticamente significativo. Para determinar el punto óptimo de corte se construyó una curva característica operativa del receptor (ROC), así como el cálculo del área bajo la curva (AUC). Por último, para cuantificar la validez de los resultados se utilizó el cálculo de la razón de verosimilitud positiva y la razón de verosimilitud negativa, y se construyó un nomograma de Fagan. Los datos fueron capturados en Microsoft Excel 2018 y posteriormente analizados en los programas SPSS v. 25 para Windows y R versión 4.0.

Todos los pacientes firmaron carta de consentimiento informado y el protocolo fue debidamente registrado ante el comité de ética y de investigación en salud de la unidad médica participante con el número R-2019-2105-036.

\section{Resultados}

De los 11 pacientes analizados, 8 (72.7\%) fueron hombres y $3(27.3 \%)$ mujeres. La variable edad
Tabla 1. Resumen de la estadística descriptiva de las variables demográficas de los pacientes

\begin{tabular}{|l|c|c|c|c|}
\hline & $\begin{array}{c}\text { Edad } \\
(\mathbf{a n ̃ o s})\end{array}$ & $\begin{array}{c}\text { Peso } \\
(\mathbf{k g})\end{array}$ & $\begin{array}{c}\text { Talla } \\
(\mathbf{m})\end{array}$ & $\begin{array}{c}\text { IMC } \\
\left(\mathbf{k g} / \mathrm{m}^{2}\right)\end{array}$ \\
\hline Promedio (\%) & 33 & 63 & 1.7 & 24 \\
\hline Desviación estándar & 11 & 14.3 & 0.09 & 4.6 \\
\hline
\end{tabular}

IMC: índice de masa corporal.

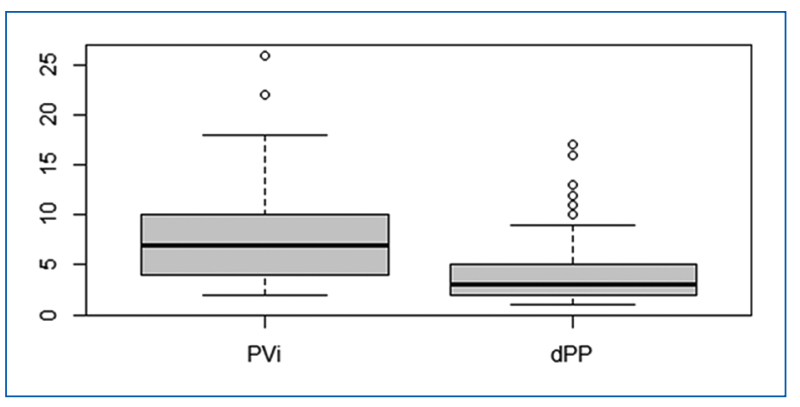

Figura 1. Muestra los brazos de las variables PVi y dPP, donde se aprecia la existencia de valores atípicos en ambas variables; 2 para PVi y 5 para dPP respectivamente. Existe un mayor rango y la dispersión es más evidente en la variable PVi.

PVi: índice de variabilidad pletismográfica; dPP: variabilidad de presión de pulso.

presenta valores comprendidos entre 25 y 65 años, con asimetría positiva (0.57) (sesgada a la izquierda) con mayoría de datos por arriba de la media aritmética y aplanamiento con curtosis -1.19 (platicúrtica), media aritmética de 33 años, DE: 11 años. Para la variable índice de masa corporal (IMC) la media aritmética es de 24.2 y la DE 4.6. El resumen de las variables demográficas se muestra en la tabla 1.

Las variablesfrecuencia cardiaca (FC) (W: $0.96 ; p=0.03)$ y presión arterial media (PAM) (W: $0.86 ; p=0.00)$ no presentan una distribución normal. Los valores de laFC fueron mediana 71.5 y RIQ de 26 latidos por minuto, y de la PAM mediana $81.5 \mathrm{mmHg}$ y RIQ de $17 \mathrm{mmHg}$.

La variable dPP presentó una mediana aritmética de $4.47 \pm 3.6$, rango 1 a 17 , y el PVi una media aritmética de $7.9 \pm 4.9$, rango 2 a 26. La diferencia entre los valores de las variables dPP y PVi fue t: 16.05 (intervalo de confianza del 95\%: 5.4-6.9; $p=0.00$ ) (se consideró estadísticamente significativo un valor de $p \leq 0.05$ ) (Fig. 1).

El polar plot muestra la existencia de una concordancia casi perfecta entre la dPP y el PVi, explicada por la presencia de la extensión de los datos cerca de 


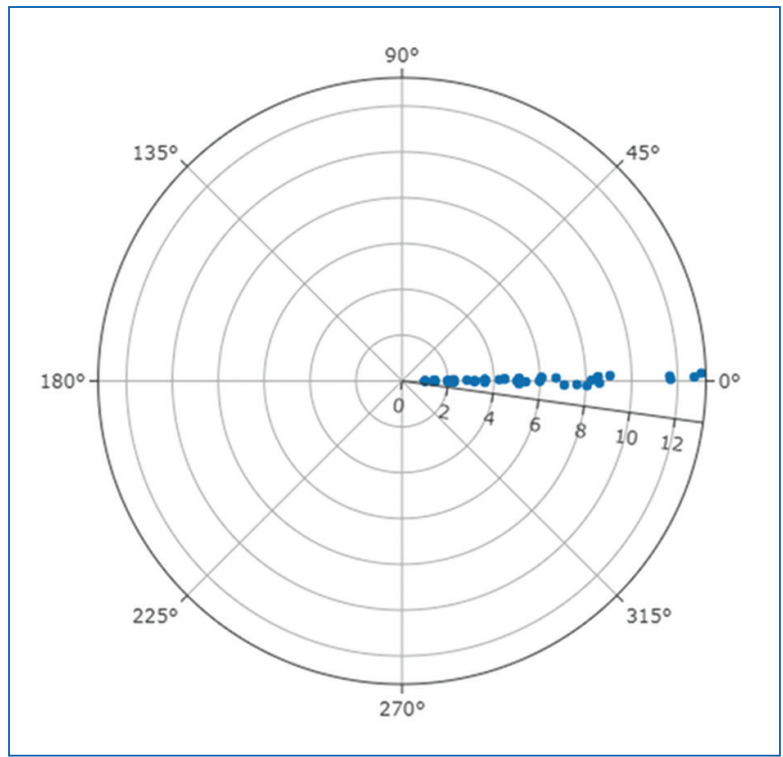

Figura 2. Polar plot para medir la concordancia de los métodos de monitoreo (variabilidad de presión de pulso e índice de variabilidad pletismográfica).

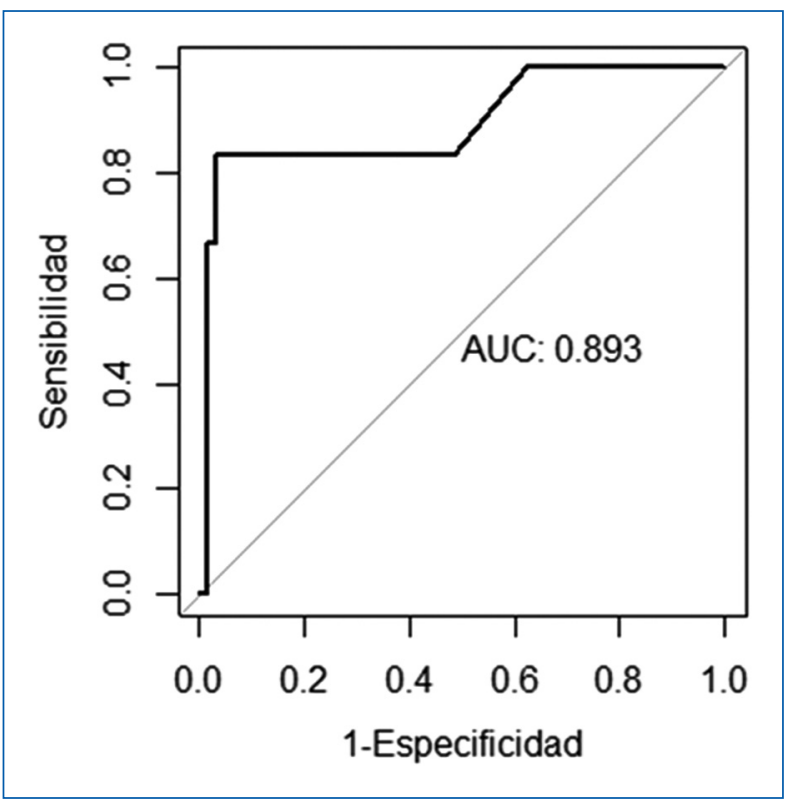

Figura 3. Característica operativa del receptor del método de prueba (índice de variabilidad pletismográfica) donde se muestra un área bajo la curva (AUC) del $89.3 \%$ para un valor de corte de 14.

la línea de referencia de la gráfica polar, ubicada a $0^{\circ}$ (Fig. 2).

Se comprobó el valor óptimo de corte para el PVi en 14; por los resultados obtenidos en la curva ROC, este punto de corte muestra una AUC del $89.3 \%$, siendo

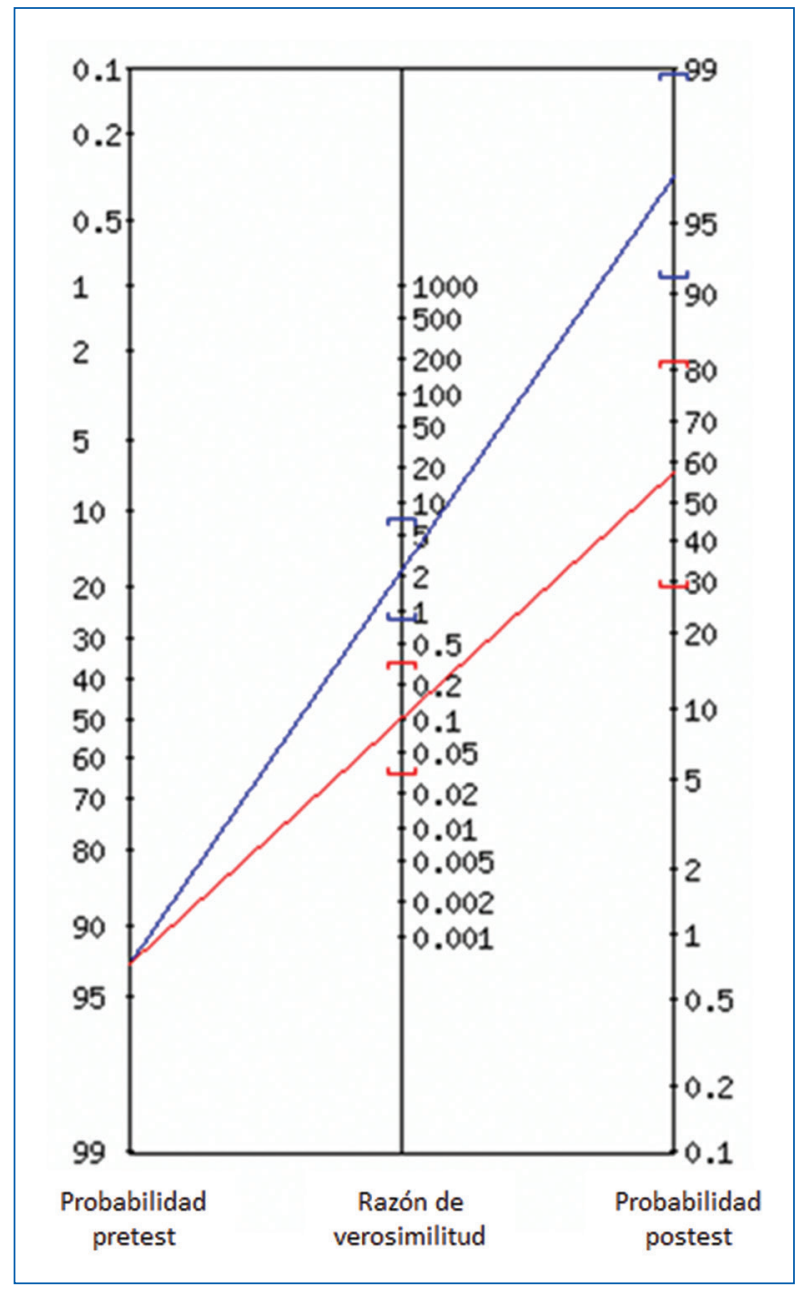

Figura 4. Normograma de Fagan. Se obtuvo una probabilidad pretest del $93 \%$, mientras que la probabilidad postest positiva fue del $97 \%$ (azul) y probabilidad postest negativa del $57 \%$ (rojo), añadiendo la razón de verosimilitud positiva de 2.35 y negativa de 0.1 .

estadísticamente significativo. Se corrobora con el cálculo de las razones de verosimilitud, las cuales fueron regular para la positiva, con 2.35 , y buena para la negativa, con 0.1 (Fig. 3).

Por último, con estos resultados y el cálculo de probabilidad pretest y postest positiva/negativa se graficó el nomograma de Fagan, que muestra una confiabilidad alta del monitoreo de prueba, al presentar una probabilidad postest positiva del $97 \%$ (Fig. 4).

\section{Discusión}

En la presente investigación se confirma que ambos métodos de monitoreo hemodinámico tienen una buena concordancia. Nuestros resultados son 
parecidos a los reportados por Addison, et al. ${ }^{11}$, quienes también reportan concordancia adecuada entre las variables dinámicas $\triangle \mathrm{POP}$ y $\mathrm{dPP}$, a pesar de que en ambas investigaciones se utilizaron metodologías diferentes: índice de correlación de Pearson con curva ROC y en nuestra investigación polar plot ${ }^{12}$. En otro estudio, Bedi, et al. ${ }^{13}$ estudiaron la concordancia entre PVi e índice de distensibilidad de la vena cava medida por ultrasonografía, demostrando buena correlación entre ambos tipos de monitoreo para la predicción de respuesta a fluidos en pacientes críticos. Incluso existe evidencia de la correlación en otras posiciones diferente a la supina. Kim, et al. reportaron que tanto el PVi como la dPP sirvieron de igual forma para la guía de la fluidoterapia en paciente sometidos a cirugía de columna $^{14}$; en este estudio se enfocaron en los resultados clínicos al clasificar a los pacientes en respondedores y no respondedores. Entonces, se cuenta con más evidencia que soporta que el PVi primero, puede sustituir el monitoreo semiinvasivo de la dPP, con la gran ventaja de ser totalmente no invasivo, y segundo, es igual de adecuado para el monitoreo en varios escenarios y posiciones del paciente (quirófano, terapia intensiva), donde se deben de obtener los mismos resultados independientemente del monitor utilizado.

Existe controversia en el punto de corte óptimo para el PVi. En el trabajo de Coeckelenbergh, et al. en pacientes sometidos a cirugía abdominal de riesgo bajo a moderado, el PVi parece guiar de igual forma la GDT en la fluidoterapia que la dPP ${ }^{15}$. En este estudio se tomó el PVi > 15, sin embargo, no se exploró el mejor punto de corte para el PVi, siendo esta la posible causa de la diferencia entre sus resultados y los nuestros en el valor del PVi, pues el objetivo se basó en la comparación de la fluidoterapia administrada ${ }^{15}$.

El utilizar el PVi es más cómodo para el paciente, al ser un monitoreo totalmente no invasivo, evitando las complicaciones relacionadas con la colocación y manipulación de una línea arterial y tal vez, aunque no existe evidencia disponible, podría ser más económico en términos de insumos, optimizando de esta manera los recursos económicos hospitalarios, siendo parte de lo que hoy en día se define como medicina de alto valor ${ }^{16}$.

\section{Conclusiones}

El PVi es equiparable a la dPP como método de monitoreo dinámico, con una buena probabilidad postest (que se traduce en el área clínica en que el $97 \%$ de los pacientes con un $\mathrm{PVi} \geq 14$ será concordante con una $\mathrm{dPP} \geq 12$ ), para la toma de decisiones en pacientes con enfermedad renal crónica sometidos a cirugía de implante renal bajo anestesia general equilibrada, con la gran ventaja de ser totalmente no invasivo y confiable para guiar la fluidoterapia.

Es importante señalar que en el presente estudio no se cuantifica el grado de respuesta a líquidos de los pacientes, el abordaje es meramente con un enfoque de tamizaje de los diferentes tipos de monitoreo dinámico disponibles. En la presente investigación se confirma que ambos métodos de monitoreo hemodinámico tienen una concordancia buena.

\section{Financiamiento}

La presente investigación no ha recibido ninguna beca específica de los sectores público, comercial o sin ánimo de lucro.

\section{Conflicto de intereses}

Los autores declaran no tener conflicto de intereses.

\section{Responsabilidades éticas}

Protección de personas y animales. Los autores declaran que para esta investigación no se han realizado experimentos en seres humanos ni en animales.

Confidencialidad de los datos. Los autores declaran que han seguido los protocolos de su centro de trabajo sobre la publicación de datos de pacientes.

Derecho a la privacidad y consentimiento informado. Los autores declaran que en este artículo no aparecen datos de pacientes.

\section{Bibliografía}

1. Miller TE, Myles PS. Perioperative fluid therapy for major surgery. Anesthesiology. 2019;130(5):825-32

2. Shoemaker WC, Appel PL, Kram HB, Waxman K, Lee TS. Prospective trial of supranormal values of survivors as therapeutic goals in high-risk surgical patients. Chest. 1988;94(6):1176-86.

3. Bennett VA, Cecconi M. Manejo de fluidos perioperatorios: desde la fisiología hasta la mejora de los resultados clínicos. Indio J Anaesth. 2017;61(8):614-21.

4. Guerrero GM, Pérez NO, Zamarrón LE, Sánchez DJ, Escarramán MD, Deloya TE, et al. Invasive, minimally invasive and non-invasive hemodynamic monitoring in perioperative medicine. $\mathrm{J}$ Anesth Crit Care Open Access. 2020;12(1):7-19.

5. Cannesson M, Aboy M, Hofer CK, Rehman M. Pulse pressure variation: where are we today? J Clin Monit Comput. 2011;25(1):45-56.

6. Perel A. The physiological basis of arterial pressure variation during positive pressure ventilation. Réanimation. 2005;3(14):162-71.

7. MASIMO Technical bulletin. Pleth Variability Index: A dynamic measurement to help assess physiology and fluid responsiveness. 2013. Consultado 02 de julio 2021. Disponible en: https://anesthesiology.queensu.ca/ sites/anesthesiology/files/inline-files/LAB4583B_Technical_Bulletin_Pleth_Variability_Index.pdf 
8. Perel A. Using dynamic variables to guide perioperative fluid management. Anesthesiology. 2020;133(4):929-35.

9. Maguire S, Rinehart J, Vakharia S, Cannesson M. Technical communication: respiratory variation in pulse pressure and plethysmographic waveforms: intraoperative applicability in a North American academic center. Anesth Analg. 2011;112(1):94-6.

10. Germán S, Luciano C, Guillermo G. Índice de Variabilidad Pletismográfica (PVi): Monitoreo continuo y no invasivo de la administración de fluidos. Novedades en tecnología. Rev Argent Anestesiol. 2013;71(2):48-58.

11. Addison PS, Wang R, Uribe AA, Bergese SD. Increasing signal processing sophistication in the calculation of the respiratory modulation of the photoplethysmogram (DPOP). J Clin Monit Comput. 2015;29(3):363-72.

12. Critchley LA, Yang XX, Lee A. Assessment of trending ability of cardiac output monitors by polar plot methodology. J Cardiothorac Vasc Anesth. 2011;25(3):536-46.
13. Bedi PS, Pahwa B, Hooda B, Dwivedi D. Plethysmographic variability index as a tool to assess fluid responsiveness in critically ill patients: a correlation study with inferior vena cava distensibility index. Int J Res Med Sci. 2019;7(12):4663-72.

14. Kim HD, Shin S, Kim JY, Kim SH, Jo M, Choi YS. Pulse pressure variation and pleth variability index as predictors of fluid responsiveness in patients undergoing spinal surgery in the prone position. Ther Clin Risk Manag. 2018;14:1175-83.

15. Coeckelenbergh S, Delaporte A, Ghoundiwal D, Bidgoli J, Fils JF, Schmartz $D$, et al. Pleth variability index versus pulse pressure variation for intraoperative goal-directed fluid therapy in patients undergoing low-to-moderate risk abdominal surgery: a randomized controlled trial. BMC Anesthesiol. 2019;19(1):34.

16. Ñamendys-Silva SA. High value care in critical care medicine. Med Crit. 2019;33(2):91-7. 